\title{
On The Performance of the RB3-LYP, RMP2 and UCCSD(T) Procedures in Calculating Radical Stabilization Energies for $\bullet \mathbf{N H X}$ Radicals
}

Geoffrey P. F. Wood, David J. Henry, and Leo Radom

Research School of Chemistry, Australian National University, Canberra, ACT 0200, Australia, and School of Chemistry, University of Sydney, Sydney, NSW 2006, Australia

\section{SUPPORTING INFORMATION (One Page)}

TABLE S1: Total Energies (Hartrees, Without ZPVEs) for the Ground States (A" or pseudo-A") of •NHX Radicals Calculated at Various Levels of Theory with the 6-31+G(d) Basis Set on UB3-LYP/6-31+G(d) Geometries

\begin{tabular}{lccccc}
\hline Level & $\bullet \mathrm{NH}_{2}$ & $\bullet \mathrm{NHCF}_{3}$ & $\bullet \mathrm{NHCHO}$ & $\bullet \mathrm{NHCOCH}_{3}$ & $\bullet \mathrm{NHCONH}_{2}$ \\
\hline UHF & -55.56010 & -391.19280 & -168.29900 & -207.34311 & -223.35585 \\
RHF & -55.55576 & -391.18826 & -168.28303 & -207.33242 & -223.35019 \\
UB3-LYP & -55.87887 & -392.94124 & -169.215762 & -208.54429 & -224.60161 \\
RB3-LYP & -55.87732 & -392.93967 & -169.21230 & -208.54136 & -224.59951 \\
UMP2 & -55.69658 & -391.97390 & -168.71486 & -207.89827 & -223.95632 \\
RMP2 & -55.69661 & -391.97409 & -168.72910 & -207.90846 & -223.95715 \\
& & & & & \\
UCCSD(T) & -55.71771 & -392.01398 & -168.76796 & -207.96341 & -224.00392 \\
URCCSD(T) & -55.71771 & -392.01397 & -168.76823 & -207.96242 & -224.00396 \\
\hline
\end{tabular}

